\title{
TOOL CONDITION MONITORING IN MACHINING - NEURAL NETWORKS
}

\author{
Mo A. Elbestawi \\ McMaster University \\ elbestaw@mcmaster.ca \\ Mihaela Dumitrescu \\ McMaster University \\ dumitrm@memaster.ca
}

\begin{abstract}
Condition monitoring and diagnosis systems capable of identifying machining system defects and their location are essential for unmanned machining Unattended (or minimally manned) machining would result in increased capital equipment utilization, thus substantially reducing the mantacturing costs. A review of tool monitoring systems and techniques and their components and the Multiple Principle Component fizzy netral network for tool condition monitoring machining are presented.
\end{abstract}

\section{INTRODUCTION}

Increased demands for even higher product quality, reliability, and manufacturing efficiency levels have imposed stringent requirements on automated product measurement and evaluation. Manufactured products of the modern day command ever-higher precision and accuracy, therefore automated process monitoring becomes crucial in successfully maintaining high quality production at low cost.

The automated tool condition monitoring processes imply the identification of cutting tool condition without interrupting the manufacturing process operation, under minimum human supervision. Unattended or minimally manned machining leads to increased capital equipment utilization, thus substantially reducing the manufacturing costs. Both these situations require intelligent sensor systems.

An "Intelligent Sensor System" was defined by (Dornfeld, 1986) ${ }^{1}$ as an integrated system consisting of sensing elements, signal conditioning devices, signal processing algorithms, and signal interpretation and decision making procedures. In the absence of a human operator, the system should sense signals indicating the process status and its changes, interpret incoming sensed information, and decide on the appropriate control action.

A system could be defined as Automated/Intelligent Monitoring System if sensing, analyzing, knowledge learning, and error correction abilities, essential to machining tool condition monitoring, are incorporated. 
An automated/intelligent machining process and tool condition monitoring system should be able to emulate as closely as possible the abilities of human operators. Thus, the following four essential components have to be included in any automated tool condition monitoring system to emulate the human monitoring action:

Sensing Technique Systems: Typically, indirect sensing techniques such as cutting forces, vibrations, and acoustic emission are used. Different types of sensors and sensory data from different locations are combined to yield maximum useful information.

Feature Extraction Systems: Ideally, sensory signals contain the necessary information required to discriminate between different process and tool conditions. However, these signals are usually noisy and require further processing to yield useful features, highly sensitive to the tool conditions but insensitive to external noises.

Decision Making Systems: Decision making strategies process incoming signal features and perform a pattern association task, mapping the signal feature to a proper class (tool condition). This processing task can be done sequentially or in parallel depending on the monitoring system architecture.

Knowledge Learning Systems: In order to make a correct decision, learning algorithms have to be provided. Such algorithms tune system parameters by observing the sample features corresponding to different tool conditions. Like human operators, automated monitoring systems should have the ability to learn from their experiences (past work) as well as from the new information generated from the machining process.

Key concerns with both signal processing and decision-making algorithms, jointly known as monitoring methods, include reliable and fast identification or response to an abnormal event occurring at normal process conditions ( $\mathrm{Du}, 1995,{ }^{2}$.)

\section{RESEARCH ISSUES}

The major research goals for tool condition monitoring are to develop self-adjusting and integrated monitoring systems able to function under various working conditions with minimum operator supervision.

The purpose of automated tool condition monitoring in machining is to relate the process signals to the tool conditions, and detect or predict tool failure. Automated tool condition monitoring implies identifying the characteristic changes of the machining process based on the evaluation of process signatures without interrupting normal operations. Basically, a monitoring process has two parts:

- Sensing- obtaining cutting process signals from sensors. Appropriate signals used for tool condition monitoring are force, torque, vibration, temperature, acoustic emission, electric current, etc...)

- Monitoring- composed of signal processing and decision making, can be divided into model-based and feature-based methods. Both methods use sensor signals from the cutting process for the system input.

Any automated machining process and tool condition monitoring systems should include: 
- "Multi-Sensor Systems" - more than one sensor should be used for monitoring machining processes and tool conditions, yielding an extended survey of sensitive features.

- "Automated Feature Extracting Systems" - automatically generate monitoring features through learning. The signals sensed from multiple sensors are analyzed, compacted, and selected by the system to yield the most sensitive features to the monitoring subjects. The extracted features are also further refined or reselected by the monitoring system.

- "Learning and Decision Making Systems" - build up flexible and comprehensive monitoring strategies and automatically generate control parameters. The concentrated information from the learning procedure is stored in the system for classification purposes and can be modified by knowledge updating procedures. With increasing experience, the system will become more and more reliable and promote the monitoring/control functions. These strategies should be robust and valid for a reasonable range of cutting conditions.

Significant research work performed in this research field focused on analytical forecast, dynamic structure identification, monitoring techniques, and adaptive control approaches; Thonshoff et.al.,1998,,; Tlusty and Andrews, 1983,; Isserman, 1984, ${ }^{5}$; and Dornfeld, 1990, ${ }^{6}$ published research papers on the development of modern monitoring techniques for machining.

Critical reviews on sensors for machining monitoring were published by Tlusty and Andrews, 1983 and Dornfeld, 1992,? Applications include geometric corrections, machine diagnosis, surface finish controls, tool condition monitoring, and machining process monitoring.

Thonshoff et.al., 1998, identified five monitoring tasks: machine, tool, process, tool condition, and workpiece; the author described the monitored conditions in machining processes and classifies the monitored functions into two groups: time critical and non-time critical. The former requires a system response within a range of milliseconds while the later may take seconds or even minutes.

\subsection{Sensing Techniques}

Metal cutting is a dynamic process; the sensor signals can be considered as the output of the dynamic system in a form of time series. Consequently, process and tool condition monitoring can be conducted based on system modeling and model evaluation. One of the most used models is linear time-invariant system, such as state space models, input-output transfer function models, Auto-Regressive (AR) models, Auto-Regressive and Moving Average (ARMA) models, and Dynamic Data Systems (DDS) methodology. When a suitable model is identified, monitoring can be performed by detecting the changes of the model parameters and/or the changes of expected system responses.

Current sensing techniques can be divided into two basic types:

- Direct Techniques - the most accurate measures for determining tooling failure; however the trade-off is production stoppage. With these methods, a direct analysis of the tool or workpiece surface is performed at the end of the machining cycle. Basic analysis methods include optical measurements, surface finish using contact probes (profilometers), chip size 
measurements, etc. The main disadvantages of this methods is that any significant deterioration occurring in between measurements goes unnoticed, allowing for potential damage to the "machine tool- toolingworkpiece" (MTW) system.

- Indirect Techniques - use correlated variables with process signals to monitor for a specific signature of tool failure. These techniques can be applied continuously while machining, and therefore can be used in an online monitoring algorithm. The most common methods able to correlate a variable with tool condition are:

- Spindle Current Monitoring

- Acoustic Emissions

- Vibration Signature Analysis

\section{Sensor Fusion}

In most cases, signals coming from only one sensor are typically insufficient to give enough information for machining and tool condition monitoring.

Using several sensors at different locations simultaneously was proposed for data acquisition by Ruokangas et.al., 1986*; McClelland, 1988'; Chryssolouris et.al., $1998^{10}, 1989^{11}$ and 1992'2; and Dornfeld, 1990. Signals from different sources are integrated to provide the maximum information needed for monitoring and control tasks. A schematic diagram of using multiple sensors in monitoring systems is shown in Figure 1.

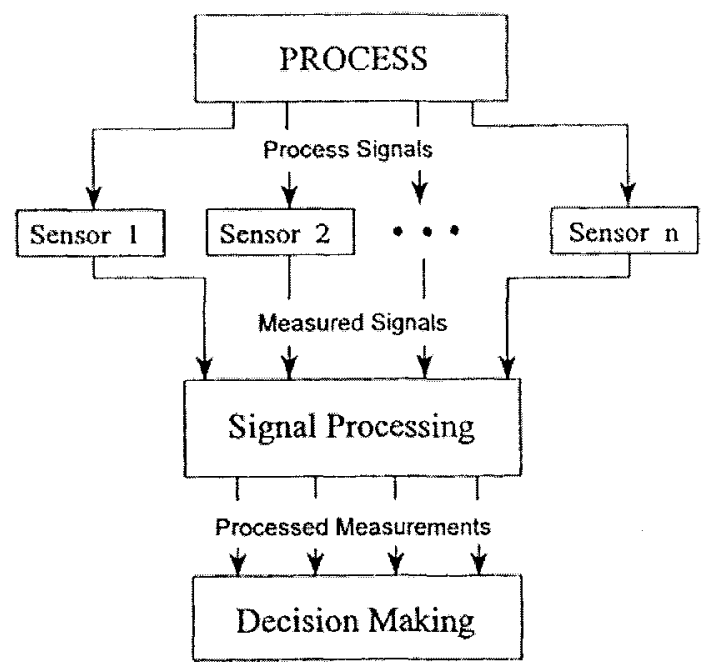

Figure 1 - Multiple Sensors in Monitoring

Sensor fusion generally covers all the issues of linking sensors of different types together in one underlying system architecture (McClelland, 1988, 9.) The strategy of integrating the information from a variety of sensors will increase the accuracy and resolve ambiguities in the knowledge about the environment. The most 
significant advantageous aspect of sensor fusion is its enriched information for feature extraction and decision making strategy, and its ability to accommodate changes in the operating characteristics of the individual sensors due to calibration, drift, failure, etc... The type and number of sensors used in tool conditioning monitoring are chosen according to the type of monitoring tasks. The most common types of sensors used in monitoring systems were identified by Tlusty, 1983.

The use of multiple sensors for machining process and tool condition monitoring gives extended information about the process. As most process variables have an influence on one another, more than one model is typically needed to analyze the sensor signals.

Some models considered are: dynamic structure models for cutting force, such as empirical cutting force model (Endres et.al, 1990, ${ }^{13}$ ), dynamic models for tool wear such as diffusion wear models, adhesive wear models, empirical models (Koren et.al., 1991, ${ }^{14}$ ), linear steady-state models for tool wear and cutting forces (Koren, 1978, ${ }^{15}$; Koren et.al, 1987, ${ }^{16}$ and Matsumoto et.al, 1988, $\left.{ }^{17}\right)$, and parametric models including Auto-Regressive methods (AR) for chatter (Yang et.al, 1982, ${ }^{18}$ and Tsai et.al, 1983, ${ }^{19}$ ), AR for tool wear ( Liang et.al, 1989, ${ }^{20}$ )], and AR for tool breakage (Takata and Sata, 1986, ${ }^{21}$.)

Emphasis on high reliability and fast response is placed on tool condition monitoring systems to ensure the manufacturing of high quality parts in an efficient manner. Early detection of tool deterioration improves process productivity and reliability. Therefore, monitoring systems must be developed with the above criteria in mind. Computing time and adaptive learning are also both important factors to consider in developing monitoring systems (Du et.al, 1995, ${ }^{22}$.)

\subsection{Feature Extraction Methods}

According to $\mathrm{Du}, 1995$, monitoring methods can be sub-divided into two basic types: model-based and feature-based methods:

Model-based methods involve finding a model that fits the process and monitoring specific parameters in that model to detect changes. Changes in the expected system response can be interpreted as changes in the process, signifying an abnormal cycle. Model based monitoring methods are also referred to as failure detection methods by Isserman et.al, 1993, ${ }^{23}$ and Lee et.al;, 1998, ${ }^{24}$.

Feature-based monitoring methods use mapping processes to identify the process and tool conditions and relate the tool conditions to the sensor features. Such techniques include pattern recognitions, expert systems, neural networks, and fuzzy classifications. The feature-based methods consist of two phases: learning and classification.

Learning, also called training, is the procedure of establishing the system structure and classification rules. The knowledge for decision making is obtained from the learning samples as well as from instructions. Knowledge updating or continuous learning implies that the system is retrained with new available information.

Monitoring tasks are done in classification phase. The structure and knowledge base built in the learning phase are used for the decision making in monitoring. 


\subsection{Decision-Making Methods}

Decision making strategies are one of the major issues in the development of automated machining process and tool condition monitoring. They range from simple threshold limit values being exceeded and triggering abnormal conditions, to systems requiring substantial training and learning to determine process characteristics of each cutting condition and using these to detect out of specification conditions. A decision making process in monitoring is based on the relationship between the process/tool conditions and the feature-bearing signals (monitoring indices).

Currently used feature-based monitoring methods include: pattern recognition, fuzzy systems; decision trees, expert systems and neural networks - which will be discussed further.

Among the large number of decision making methods that have been developed, statistical pattern recognition, neural networks and fuzzy classification are very interesting aspects in the development of automated/intelligent tool condition monitoring in machining. They have been applied successfully in many cases of monitoring tasks in turning, milling, drilling, and other metal cutting processes.

The pattern recognition technique has been applied to recognize the cutting states and to monitor the tool conditions in machining for decades. The most simple and popular algorithms use linear classifiers.

A linear model was used in applications of linear classifiers by Zhang et.al, $1982,{ }^{25}$; Marks and Elbestawi, 1988, ${ }^{26}$; and Liu et.al, 1988, ${ }^{27}$.

The features for classifying the cutting states included cutting speed, feed and the power spectrum in different frequency bands.

Features usually used for tool condition monitoring are: feed rate, depth of cut, cutting force, cutting torque, and the sum of the magnitudes of spectral components at certain frequencies. Experiments showed that the number of features and the different combinations of features had great effects on the correct classification rates. The success rate of classification with these cases is $77 \%$ or higher.

Other pattern recognition algorithms for tool condition monitoring in machining included the class-mean scatter criterion, the class variance criterion, and Fisher's weighted criterion (Emmel et.al. 1987, ${ }^{28}$, and $1988,{ }^{29}$.) The class-mean scatter criterion maximizes class separation and minimizes within-class variance. The class variance criterion maximizes the difference between the within-class variance of each class. Fisher's weight criterion maximizes class separation and minimizes the within-class variance between each pair of classes. This methodology was applied in order to detect tool wear and breakage in turning operations using acoustic emission spectral information under fixed cutting conditions. The tool wear sensing results had performances ranging from 84 to $94 \%$.

\section{NEURAL NETWORKS FOR TOOL CONDITION MONITORING}

Neural networks are computing systems made up of a number of simple, highly interconnected processing elements that provide the system with the capability of self-learning. Using neural networks, simple classification algorithms can be used 
and the system parameters are easily modified. One major characteristic of building neural networks is the training time. Training times are typically longer when complex decision regions are required and when networks have more hidden layers. As with other classifiers, the training time is reduced and the performance improved if the size of a network is optimally tailored. The tasks of an automated tool condition monitoring system involve the ability to recognize the tool condition by analyzing measured cutting process parameters such as forces and vibrations. This ability is based on the accumulation of useful information from related laws of physics and operators' experiences. In building automated/intelligent tool condition monitoring systems, some basic functions have to be considered:

1. Fusion of multiple sensors;

2. Learning or training strategies for the monitoring system;

3. Knowledge updating techniques; and

4. Description of the imprecision in tool conditions for various cutting conditions.

With the increasing needs for effective and robust automated machining process and tool condition monitoring, a significant amount of research work has been performed to find decision making strategies. The principal constituents of soft computation include fuzzy logic for imprecision in the acquired data, neural networks for learning, and probability reasoning for uncertainty. These three components are usually overlapped. The "soft computation" is easily implemented by fuzzy neural networks.

\subsection{Multiple Principal Component (MPC) Fuzzy Neural Networks Structure}

Learning refers to the processes which build the monitoring system in a given structure with information from the learning data. In addition, some logic rules are also created, which determine the data processing and govern the relationship between the processing elements. During the learning phase, a limited amount of data is used to adjust the parameters of the monitoring system. The trained monitoring system uses the stored knowledge to classify the data regarding the successive tool conditions.

If the sampled data for training the system are labeled with the class to which a sample belongs, the decision making is performed with a priori knowledge. This is called pattern classification, or supervised classification, common in automated tool condition monitoring in machining. When the training samples are collected, the tool conditions related to each training sample are provided to give the necessary information.

Knowledge updating, or self-learning, essential for an automated tool condition monitoring system, refers to processes in which the structure and the parameters of a monitoring system are modified according to the new information about the classification. Classification results should be checked on-line to ensure the system gives correct results. If the results are not correct, the system should be retrained or modified. 


\subsection{Construction of the MPC Fuzzy Neural Networks}

The Multiple Principal Component (MPC) Fuzzy Neural Networks are constructed based on the idea of "soft computation." Neural networks, fuzzy logic and statistical reasoning are employed. Simple classification procedures can be implemented at individual processing elements (neurons). The interconnections between neurons in the network communicate the information and make it possible to solve complex classification problems. Statistical reasoning is used in the learning procedure for the feature extraction and the partition strategies.

For conventional neural networks, each of the processing elements (for input, output, and hidden layers) is always connected to every single processing element in the neighboring layers. Decision tree classifiers are hyperplane classifiers that can be regarded as a type of partially connected neural networks since each node in the tree is connected to only its "father" and "sons", requiring comparatively less classification computations and can be implemented using parallelism from decision region by performing simple, easily understood operations on the neural network. In more sophisticated implementations, multi-layered neural networks, consisting of nonlinear connections between the inputs and the outputs are employed.

As an alternative to conventional neural networks, a partially connected, fuzzy neural network approach can be used for automated tool condition monitoring in machining. Different from matrix-type decision making, a tree structure is used for reducing unnecessary connections between elements in the input and the output layer. The fuzzy classifications are used in the neural networks to provide a comprehensive solution for certain complex problems. The neural network that utilizes fuzzy classification is shown in Figure 2.

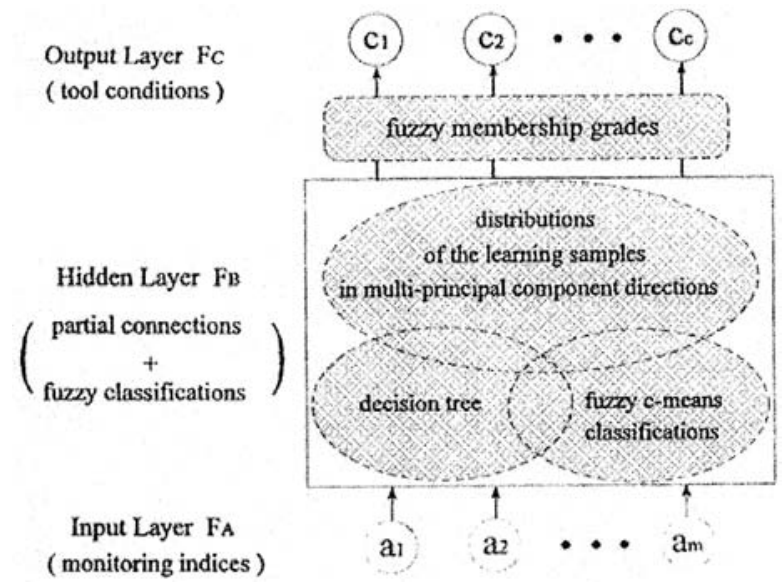

Figure 2 - The Multiple Principal Component (MPC) fuzzy Neural Network

The input layer, $F A=\left(a_{1}, a_{2}, \ldots, a_{m}\right)$, has $m$ processing elements, one for each of the $m$ dimensions of the input pattern $x_{1 n}$. The hidden layer of the network, FB, consists of the neurons that use the fuzzy classification to separately address the subsets of 
the original data set while invoking necessary information from other neurons. The probability distribution and the membership function are used for interconnections within the hidden layer and the connections to the output layer. The neurons of the output layer, FC, represent the degrees to which the input pattern xk fits within the each class. There are two possible ways that the outputs of FC class nodes can be utilized. For a soft decision, outputs are defined with the fuzzy grades. For a hard decision, the FC nodes with the highest membership degree are located (a "winnertake-all" response). The connections within the hidden layer are not from one element to every one in the neighboring layers. The structure depends on the training data and is created through the training process. These partial connections result in the simpler and faster training and classification.

\subsection{Evaluation of MPC Fuzzy Neural Networks}

A pattern classifier should possess several properties: on-line adaptation, nonlinear separability, dealing with overlapping classes, short training time, soft and hard decisions, verification and validation, tuning parameters, and nonparametric classification (Simpson, $1992,{ }^{30}$.) The MPC fuzzy neural networks for automated tool condition monitoring has been developed in consideration of these requirements. The training and classification algorithms are based on the theories of neural networks, fuzzy logic, and probability reasoning.

With the application of fuzzy classification, the neural networks are effective in dealing with nonlinear separable and/or class-overlapping classification problems, which are common in the case of tool condition monitoring in machining, especially for the monitoring with varying cutting conditions. The partial interconnections within the fuzzy neural networks make the training time very short compared to that of fully connected networks such as the back-propagation neural networks. The calculations necessary for the classification are also significantly reduced since not all the neurons in the hidden layer are used while a sample is being processed. Soft and hard decisions are optional for different applications. The maximum partition algorithm is based on the distributions of the learning samples and no parameter estimations are needed.

This method functions similarly, in the partition of training samples, to the linear fuzzy equation algorithm proposed by Du and Elbestawi, 1992, ${ }^{31}$. The linear fuzzy equation method uses a matrix to describe the relationship between the monitoring indices and the tool conditions. The proposed MPC fuzzy neural networks use a tree structure similar to that in the fuzzy decision tree described by $\mathrm{Li}$ et.al.,1992, ${ }^{32}$. Because the decision tree is more flexible than a matrix approach, it has better performance in the case of tool condition monitoring in machining. In constructing the fuzzy decision tree, the maximum partition generates nodes holding the samples from only one tool condition. The other samples are put into other nodes. This means each partition leads to a final decision at a leaf node of the tree. The maximum partition in the MPC fuzzy neural networks chooses a better partition so that a new-born neuron can hold samples from different tool conditions. A neuron can lead to other neurons in the hidden layer as well as neurons in the output layer.

The consequence of this structure is simplicity in the interconnection and the short routines in the classification. Experimental tests by using the same set of data 
showed that the MPC fuzzy neural networks gave a better success rate than the fuzzy decision tree algorithm ( $\mathrm{Li}$ and Elbestawi, 1994, ${ }^{33}$.)

In the consideration of on-line adaptation (on-line learning) and the tuning parameters, a classifier should have as few parameters to tune in the system as possible. Both the back-propagation neural networks and the proposed MPC fuzzy neural networks have very few tuning parameters. The structure of the MPC fuzzy neural network is, however, easily modified with new learning samples. Unlike the back-propagation neural networks that require a complete retraining of the system with both the old and the new learning data, the MPC fuzzy neural networks need only to change partially their neurons and the connections when the new learning information is added. Both supervised and unsupervised classification algorithms are easily implemented with the available learning samples.

To insure a good distribution of the learning data, the training samples have to be representative of the whole span of the feature space. In tool condition monitoring, all applicable tool and cutting conditions have to be considered during the training phase. On the other hand, if a poor distribution is encountered, then a modified feature extraction procedure has to be implemented. Information about new phenomena can be added to the monitoring system by knowledge updating.

\subsection{Fuzzy Classification and Uncertainties in Tool Condition Monitoring}

During machining, cutting conditions (e.g., cutting speed, feed, depth of cut) as well as tool conditions (e.g., tool wear) significantly affect the process parameters such as cutting forces and vibrations, which are usually used as the input signals to a monitoring system. Deterministic models which attempt to describe the relationship between the tool conditions and the various measured parameters are typically valid for a limited range of cutting conditions. The fuzzy classification can be used to describe the uncertainties and the overlapped relationship of the tool conditions and the monitoring indices. Briefly, the fuzzy expression of a tool condition, A, can be defined by:

$$
A=\left\{x \mid \mu_{A}(x)\right\}
$$

where $x$ is the value of $\mathrm{A}$, and $\mu_{\mathrm{A}}(x)$ is a fuzzy measure, also known as the membership function. $\mu_{\mathrm{A}}(x)$ is a monotonous function, and $0 \leq \mu_{\mathrm{A}}(x) \leq 1$. The function increases with respect to the decrease of the uncertainty of $A$. If $B$ is also a fuzzy set and is more uncertain than $A$, then:

$$
\mu_{\mathrm{A}}(x)>\mu_{B}(x)
$$

This might be interpreted as "the membership grade of small tool wear is greater than that of large tool wear." The fuzzy representation of the tool conditions in machining has its advantages. The concept of fuzzy decision making in machining tool condition monitoring is illustrated in Figure 3, where, $A_{H}$ and $B_{H}$ are categories classified by the hard decision, while $A_{F}$ and $B_{F}$ are classified by the fuzzy decision. 


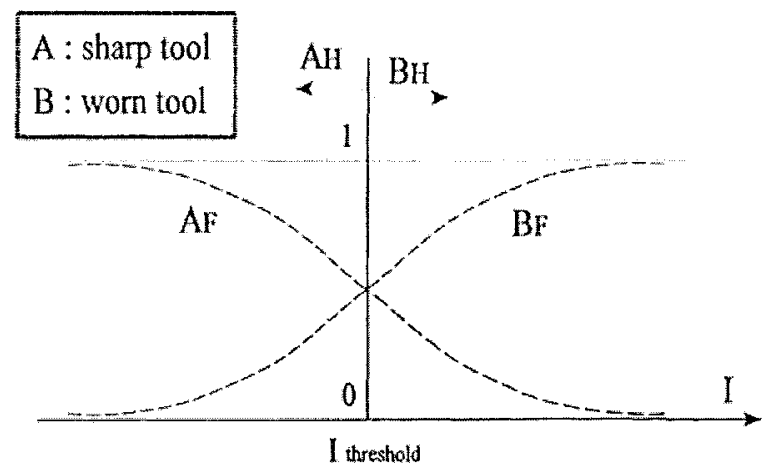

Figure 3 - Soft Boundaries in Fuzzy Classification

\section{CONCLUSIONS}

Three major components of the "soft computation" are involved in the construction of theMPC Fuzzy Neural Networks. The combination of fuzzy logic with neural networks has a sound technical basis because these two techniques approach the design of intelligent machines from different angles. Fuzzy neural networks employ the advantages of both neural networks and fuzzy logic. Neural networks offer good performance in dealing with sensor information in parallel at a low computational level. The high interconnection within the networks gives the capabilities of exchanging the information sufficiently and managing nonlinearity. Fuzzy logic gives a means for representing, manipulating, and utilizing the data and the information that possess non-statistical uncertainties.

\section{REFERENCES}

1. Dornfeld, D. A., 1986, "Acoustic Emission Monitoring for Untended Manufacturing," Proceedings. of Japan/USA Symposium. on Flexible Automation, JAACE.

2. Du, R., Elbestawi, M. A. and Wu, S. M., 1995, "Autonated Monitoring of Manufacturing Processes, Part 1: Monitoring Methods," ASME J. of Eng. for Industry, Vol.117, pp. 121-132.

3. Tonshoff, H. K. and Wulfsberg, J. P., 1988, "Developments and Trends in Monitoring and Control of Machining Processes," Annals of the CIRP, Vol.37, No.2, pp. 611-622.

4. Tlusty, J. and Andrews, G. C., 1983, "A Critical Review of Sensors for Unmanned Machining," Annals of the CIRP, V 01.32, No.2, pp. 563-572.

5. Iserrmann, R., 1984, "Process Fault Detection Based on Modeling and Estimation Methods -A Survey," Automatica, Vol.20, No.4, pp. 387-404.

6. Dornfeld, D. A., 1990, "Neural Network Sensor Fusion for Tool Condition Monitoring," Annals of the CIRP, Vol.39, No.1, pp. 101-105.

7. Dornfeld, D. A., 1992, "Monitoring of Machining Process -Literature Review," Presented at CIRP STC"C" Meeting, Paris, France.

8. Ruokangas, C. C., Blank, M. S., Matin, J. F. and Schoenwald, J. S., 1986, "Integration of Multiple Sensors to Provide Flexible Control Strategies," Proceedings of the 1986 IEEE International Conference on Robotics and Automation, pp. 947-953.

9. McClelland, S., 1988, "Tacking the Problem of Sensor Fusion," Sensor Review, Vol.8/No.2, pp. 89-91. 
10. Chryssolouris, G. and Domroese, M., 1988, "Sensor Integration for Tool Wear Estimation in Machining," Sensors and Control for Manufacturing, ASME PED-Vol. 33, pp. 115-123.

11. Chryssolouris, G. and Dornroese, M., 1989, "An Experimental Study of Strategies for Integrating Sensor Information in Machining," Annals of the CIRP, Vol. 38, No. I, pp. 425- 428.

12. Chryssolouris, G., Domroese, M. and Beaulieu, P., 1992, "Sensor Synthesis for Control of Manufacturing Process," ASME J. of Engineering for Industry, Vol.114, pp. 158-174.

13. Endres, W. J., Sutherland, J. W., DeVor, R. E. and Kapoor, S. G., 1990, "A Dynamic Model of the Cutting Force System in the Turning Process," Monitoring and Control for Manufacturing Process, ASME PED. Vol.44, pp. 193-212.

14. Koren, Y., Ko, T. R., Ulsoy, A. G. and Danai, K., 1991, "Flank Wear EstimationUnder Varying Cutting Conditions," ASME J.of Engineering for Industry, Vol.113, pp. 300-307.

15. Koren, Y., 1978, "Flank Wear Model of Cutting Tools Using Control Theory," ASME Journal of Engineering for Industry, paper No. 77-WA/PROD-20.

16. Koren, Y., Danai, K., Ulsoy, A. G. and Ko, T. R., 1987, "Monitoring Tool Wear Through Force Measurement," Proceedings of the 15th NAMRI Conference, pp. 463-468.

17. Matsumoto, Y., Tjinag, N. and Foote, B., 1988, "Tool Wear Monitoring Using A Linear Extrapolation," Sensors and Control for Manufacturing, ASME-PED Vol. 33, pp.83-88.

18. Yang, W. Q., Hsieh, S. H. and Wu, S. M., 1982, "Adaptive Modelling and Characterization for Machining Chatter," Measurement and Control for Batch Manufacturing, ASME, pp. 135-144.

19. Tsai, S. Y., Eman, K. F. and Wu, S. M., 1983, "Chatter Suppression in Turning," Proceedings of the 11 th NAMRC, pp. 399-402.

20. Liang, S. Y. and Dornfeld, D., 1989, "Tool Wear Detection Using Time Series Analysis of Acoustic Emission," ASME J Journal of Engineering for Industry, Vol.11 1,.pp. 199-205.

21. Takata S. and Sata, T., 1986, "Model Referenced Monitoring and Diagnosis Application to the Manufacturing System," Computers in Industry, Vol. 7, pp. 31-43

22. Du, R., M.A. Elbestawi, and S. Wu (1995, May). Automated Monitoring of Manufacturing Processes, Part 2: Applications. Journal of Engineering for Industry, Vol. 117, pp.133-141

23. Isermann, R., M. Ayoubi, H. Konrad, and T. Reib (1993). Model Based Detection of Tool Wear and Breakage for Machine Tools. Proceedings of the IEEE International Conference System Manufacturing Cybernetics Vol. 3, pp72-77

24. Lee, B.Y., H.S. Liu, and Y.S. Tarng (1998). Modeling and Optimization of Drilling Process. Journal of Material Processing Technology Vol.74, pp.149-157

25. Zhang, Y. Z., Liu, Z. F, Pan, L. X., Liu, Y. J. and Yang, W. B., 1982, "Recognition of the Cutting States for the Difficult-to-Cut Materials Application of the Pattern Recognition Technique," Annals of the CIRP, Vol.31, No.1, pp. 97-101

26. Marks, J. and Elbestawi, M. A., 1988, "Development of Machine Tool Condition Monitoring System Using Pattern Recognition," Sensors and Controls for Manufacturing, ASME-PED- Vol.33, pp. 8998.

27. Liu, T. I. and Wu, S. M., 1988, "On-Line Drill Wear Monitoring," Sensors and Control for Manufacturing, ASME-PED-Vol. 33, pp. 99-104.

28. Emmell, E. and Kannatey-Asibu, E. Jr., 1987, "Tool Failure Monitoring in Turning by Pattern Recognition Analysis of AE Signals," Sensors for Manufacturing, ASME-PED. Vol.3D, pp. 39-57

29. Emmell, E. and Kannatey-Asibu, E. Jr., 1988, "Tool Failure Monitoring in Turning by Pattern Recognition Analysis of AE Signals," ASME J. of Engineering for Industry, Vol.112, pp. 137-145

30. Simpson, P. K., 1992, "Fuzzy Min-Max Neural Networks -Part 1: Classification," IEEE Transactions on Neural Networks, Vol. 3, No.5, pp 776-786.

31. Du, R. X., Elbestawi, M. A. and Li, S., 1992, "Tool Condition Monitoring in Turning Using Fuzzy Set Theory," International Journal of Machine Tool and Manufacturing, Vol. 32, No.6, pp. 781-796.

32. Li, S., Elbestawi. M. A. and Du, R. X., 1992, "A Fuzzy Logic Approach for Multi- Sensor Process Monitoring in Machining," Sensors and Signal Processing for Manufacturing, ASME PED-Vol. 55, pp. 1-16.

33. Li, S. and Elbestawi, M. A., 1994, "Tool Condition Monitoring in Machining by Fuzzy Neural Networks," Dynamic Systems and Control, ASME-DSC. Vol. 55-2, pp. 1019- 1034. 